\title{
Correction to: Association of rheumatoid arthritis-related autoantibodies with pulmonary function test abnormalities in a rheumatoid arthritis registry
}

\author{
Sicong Huang ${ }^{1,2}$ - Xintong He ${ }^{1}$. Tracy J. Doyle ${ }^{2,3}$ - Alessandra Zaccardelli ${ }^{1}$ - Allison A. Marshall ${ }^{1,4}$. \\ H. Maura Friedlander ${ }^{1}$. Rachel B. Blaustein ${ }^{1}$. Elisabeth A. Smith ${ }^{1}$ - Jing Cui ${ }^{1}$. Christine K. Iannaccone ${ }^{1}$. \\ Taysir G. Mahmoud ${ }^{1}$ - Michael E. Weinblatt ${ }^{1,2}$ • Paul F. Dellaripa ${ }^{1,2}$ - Nancy A. Shadick ${ }^{1,2}$ • Jeffrey A. Sparks ${ }^{1,2}$ (D)
}

Published online: 4 February 2020

(C) International League of Associations for Rheumatology (ILAR) 2020

$$
\text { https://doi.org/10.1007/s10067-019-04733-9 }
$$

The publisher regrets that the two sections under the Results omitted inadvertently on the original published version of the above article. The text are as follows:

\section{Pulmonary function test results}

Among the subjects included in the study, 188 subjects had PFTs performed for clinical indications. Seropositive subjects had lower median \% predicted FEV1 80\%, IQR 59-93\%) compared with seronegative subjects $(89.5 \%$, IQR 70-101\%; p = 0.034). While there were no statistical differences between seropositive and seronegative RA for FVC, FEV1/FVC, or DLCO, the seropositive subjects tended to have lower results (Table 2). Any PFT abnormality was observed in 84 seropositive subjects and 16 seronegative subjects.

The online version of the original article can be found at https://oi.org/ 10.1007/s10067-019-04733-9

Sicong Huang

shuang@bwh.harvard.edu

$\triangle$ Jeffrey A. Sparks

jsparks@bwh.harvard.edu

1 Division of Rheumatology, Immunology and Allergy, Brigham and Women's Hospital, 60 Fenwood Road, Boston, MA 02115, USA

2 Harvard Medical School, 25 Shattuck Street, Boston, MA 02115, USA

3 Division of Pulmonary and Critical Care Medicine, Brigham and Women's Hospital, 75 Francis Street, Boston, MA 02115, USA

4 Tufts School of Medicine, 145 Harrison Avenue, Boston, MA 02111, USA
RA-related autoantibodies and any pulmonary function test abnormality

Table 3 shows the unadjusted and multivariableadjusted as sociations of RA serostatus with the primary composite outcome of any PFT abnormality (restriction, obstruction, or diffusion abnormality) on clinically indicated PFTs. Seropositivity was significantly associated with increased odds of any PFT abnormality (OR 2.29, 95\% CI 1.30-4.03), adjusted for age, sex, education, smoking status, BMI, and RA duration. Combined $\mathrm{CCP}$ and RF positivity had multivariable OR of 1.67 (95\%CI 1.03-2.69) and 1.99 (95\% CI 1.21-3.27), for any PFT abnormality compared with $\mathrm{CCP}$ and RF negativity, respectively. There was no statistically significant dose effect of CCP titer for the primary outcome ( $\mathrm{p}$ for trend 0.061). However, the RF titer dose effect had a statistically significant $\mathrm{p}$ for trend at 0.001 , such that high-titer RF was associated with two-fold increased odds for any PFT abnormality (OR 2.29, 95\%CI 1.37-3.84) compared with $\mathrm{RF}-$. In subjects with discordant $\mathrm{CCP} / \mathrm{RF}$ patterns, $\mathrm{CCP}-/ \mathrm{RF}+$ tended to have a stronger association with any PFT abnormality (OR 2.78, 95\% CI 1.20-6.44) than $\mathrm{CCP}+/ \mathrm{RF}-(\mathrm{OR} 1.91,95 \% \mathrm{CI}$ 0.75-4.87), when compared with $\mathrm{CCP}-/ \mathrm{RF}^{-}$. 
Table 2 Pulmonary function test results overall and by serologic status, among the subset of subjects who had testing performed through routine clinical care $(n=188)$

\begin{tabular}{|c|c|c|c|c|}
\hline & All $(n=188)$ & Seropositive $(n=146)$ & Seronegative $(n=42)$ & $p$ value \\
\hline $\mathrm{FEV}_{1}(\%$ predicted $)$ & $81.5(61-96)$ & $80(59-93)$ & $89.5(70-101)$ & 0.034 \\
\hline FVC (\% predicted) & $84(66-97)$ & $82.5(63-96)$ & $88(75-105)$ & 0.055 \\
\hline $\mathrm{FEV}_{1} / \mathrm{FVC}$ & $76(68-80.5)$ & $76(68-81)$ & $76.5(72-80)$ & 0.352 \\
\hline $\mathrm{D}_{\mathrm{LCO}}(\%$ predicted $)$ & $71(53-85)$ & $68(53-82)$ & $78(58-90)$ & 0.060 \\
\hline
\end{tabular}

Table 3 Unadjusted and multivariable odds ratios for any clinically-indicated pulmonary function test abnormality (restriction, obstruction, or diffusion abnormality) according to RA-related autoantibody status $(n=1272)$

\begin{tabular}{|c|c|c|}
\hline & Unadjusted OR (95\%CI) & Multivariable* adjusted OR $(95 \% \mathrm{CI})$ \\
\hline Seronegative & 1.00 (REF) & 1.00 (REF) \\
\hline Seropositive & $2.44(1.41-4.23)$ & $2.29(1.30-4.03)$ \\
\hline CCP negative & 1.00 (REF) & $1.00(\mathrm{REF})$ \\
\hline $\mathrm{CCP}$ positive & $1.87(1.18-2.96)$ & $1.67(1.03-2.69)$ \\
\hline RF negative & 1.00 (REF) & 1.00 (REF) \\
\hline RF positive & $2.18(1.35-3.52)$ & $1.99(1.21-3.27)$ \\
\hline $\mathrm{CCP}$ negative & 1.00 (REF) & 1.00 (REF) \\
\hline $\mathrm{CCP}>1$ to $\leq 3 \mathrm{x}$ ULN & $1.88(0.95-3.70)$ & $1.78(0.89-3.57)$ \\
\hline CCP $>3 x$ ULN & $1.86(1.16-3.00)$ & $1.64(1.00-2.69)$ \\
\hline$p$ for trend & 0.012 & 0.061 \\
\hline RF negative & 1.00 (REF) & 1.00 (REF) \\
\hline $\mathrm{RF}>1$ to $\leq 3 \mathrm{x}$ ULN & $1.30(0.67-2.55)$ & $1.32(0.67-2.62)$ \\
\hline RF $>3 x$ ULN & $2.60(1.58-4.27)$ & $2.29(1.37-3.84)$ \\
\hline$p$ for trend & $<0.001$ & 0.001 \\
\hline $\mathrm{CCP}-/ \mathrm{RF}-$ & 1.00 (REF) & 1.00 (REF) \\
\hline $\mathrm{CCP}+\mathrm{RF}-$ & $1.81(0.72-4.53)$ & $1.91(0.75-4.87)$ \\
\hline $\mathrm{CCP}-/ \mathrm{RF}+$ & $2.53(1.11-5.75)$ & $2.78(1.20-6.44)$ \\
\hline $\mathrm{CCP}+/ \mathrm{RF}+$ & $2.52(1.44-4.41)$ & $2.28(1.27-4.07)$ \\
\hline
\end{tabular}

*Adjusted for age (continuous, years), sex (male, female), education (less than college degree, college degree or higher), smoking status (never, past, current), body mass index (continuous, $\mathrm{kg} / \mathrm{m}^{2}$ ), and RA duration at study baseline (continuous, years) 